\title{
sur la gestion et les finances publiques Spécial COVID-19 en Mai 2020
}

\section{BUdget dE L'ÉTAT ET DES OPÉRATEURS}

\section{La préparation du budget 2021}

Une circulaire relative aux « conférences de budgétisation " pour l'élaboration du projet de loi de finances pour 2021 a été publiée le 12 mai. Le texte, signée de la directrice du budget par délégation du ministre de l'action et des comptes publics, précise que l'objet des conférences budgétaires sera, d'une part, d'arrêter définitivement I'ensemble des sous-jacents chiffrés du budget 2021, d'autre part, de tenir compte des engagements pris depuis l'élaboration du budget triennal. Les plafonds de crédits et les mesures budgétaires feront ensuite l'objet d'arbitrages de la part du Premier ministre d'ici le début du mois de juillet, dans des lettres-plafonds servant de base aux conférences de répartition. Les conférences budgétaires tiendront compte systématiquement des mesures des plans de relance. Enfin, les ministres devront faire des propositions pour recentraliser sur le budget de l'État l'ensemble de moyens extra-budgétaires (comptes d'affectation spéciale, recettes affectées, fonds sans personnalité juridique, dépenses fiscales) et pour réduire le nombre de taxes à faible rendement.

\section{Le suivi de l'impact de la crise par la commission des finances du Sénat}

La commission des finances du Sénat a continué de publier ses notes de conjoncture sur le suivi des mesures d'urgence les 4, 11 et 19 mai. Elles donnent le détail chiffré de l'impact de la crise sur les finances de l'État, de la sphère locale et de la sphère sociale. La première note du 4 mai fait notamment le bilan des suites des deux lois de finances rectificatives du 23 mars et du 25 avril 2020. La deuxième note du 11 mai évalue les conséquences de la crise sanitaire sur les recettes fiscales (-3 Md€ de TVA brute sur les quatre premiers mois et -2,8 Md€ d'Impôts sur les sociétés sur les trois premiers mois). Enfin, la note du 19 mai consacre plus de développement aux réponses européennes (avec une annexe sur les mesures d'urgence en Allemagne).

Fitch place la France en perspective négative

L'agence de notation a maintenu le 15 mai la note $A A$ qui permet à la France d'emprunter à un taux proche de zéro sur les marchés financiers. Mais, elle a abaissé la perspective à «négative " compte tenu de la forte dégradation des finances publiques et de la conjoncture économique. L'agence souligne que le ratio de dette de la France était plus élevé que celui des pays comparables dès avant la crise sanitaire et que la consolidation budgétaire est peu soutenue depuis la crise de 2008.

\section{La mobilisation des fonds européens face à la} crise

Le 20 mai, au cours d'une réunion de coordination, l'État et les régions se sont engagés à simplifier les modalités d'utilisation des fonds européens pour qu'ils bénéficient au plus grand nombre dans cette situation de crise économique exceptionnelle. Cette démarche s'est réalisée dans le cadre de la négociation entamée par l'État français et les régions avec la Commission européenne pour 2021-2027. Par ailleurs, l'État a communiqué la clef de répartition de la future enveloppe "France » (qui est estimée à $39 \mathrm{Md}$ ) pour déterminer la composition des futures enveloppes régionales. Un effort de simplification pour les porteurs de projets est annoncé.

\section{FinanCes locales}

Les mesures de soutien à la trésorerie des collectivités

Une circulaire du Premier ministre du 5 mai précise, à l'intention des préfets et des directeurs régionaux des finances publiques, la position à tenir par rapport aux collectivités en situation financière délicate. Ce texte rappelle le rôle par- 
ticulier du réseau d'alerte des comptables pour déceler les collectivités qui pourraient rapidement se trouver dans une posture fragile. Des versements anticipés de fiscalité locale ou en matière de DGF pourront être effectués. Les comptables devront mener une analyse spécifique. Par ailleurs, la circulaire rappelle que d'autres versements pourront être anticipés comme en matière de dotation d'équipement des territoires ruraux (DETR) ou de FCTVA.

\section{L'impact de la crise sanitaire sur les finances des grandes villes}

France Urbaine se livre dans une note du 12 mai à une tentative d'anticipation de l'impact financier du Covid sur les finances des grandes villes, communautés et métropoles. Dans l'immédiat, la trésorerie et le non-engagement de certaines dépenses leur permet de faire face aux surcroîts importants de dépenses ou baisses de redevances liées à la crise sanitaire. Les dépenses supplémentaires, sont chiffrées par les collectivités entre $0,2 €$ et près de $9 €$ par habitant. Dans le champ social, le surcoût engendré varie entre 1,20 € et $2,3 €$ par habitant. Le soutien aux associations oscille entre $1,3 €$ et près de $14 €$ par habitant. À titre d'exemple, Bordeaux Métropole a fait le choix de créer un fonds à hauteur de $10 \mathrm{M€}$. A la suite de la loi de finances rectificative du 25 avril 2020, la collectivité peut verser une prime pour les agents mobilisés durant cette crise, son impact budgétaire est pour l'instant non chiffré. A l'inverse, la fermeture de nombreux sites et services publics locaux (accueil du public, bibliothèques, musées,...) se traduit par une diminution des charges générales estimées entre 0 à $-12 €$ par habitant. Par ailleurs, certains investissements lourds de transports en commun en site propre pourraient être reportés voire abandonnés comme à Nancy (construction d'une ligne de tramway en suspens) ou à Nantes (renforcement des dessertes et amélioration de l'intermodalité reportée).

\section{L'impact sur les finances des départements}

Le Sénat a publié le 20 mai une sixième note sur la conjoncture et le suivi du plan d'urgence face à la crise sanitaire. Au niveau des collectivités, le principal enseignement concerne la prise en compte par la Haute assemblée des données chiffrées produites le 7 mai 2020 par l'Assemblée des Départements de France (ADF). Ceux-ci pourraient connaitre, entre 2020 et 2022, une diminution de leurs recettes équivalente à 6,1 Md€ avec une diminution dès 2020 de $4 \mathrm{Md}$. Les recettes de taxe foncière et de CVAE devraient demeurer stables en 2020, mais pour la CVAE, celle-ci pourrait connaitre une contraction de -15 à - $25 \%$ à partir de 2022. Dans le même temps, on peut s'attendre à une forte hausse des dépenses de RSA, le rapport entre les dépenses de RSA et le montant des recettes de fonctionnement pouvant excéder $25 \%$. Selon un communiqué du 25 mai 2020 de L'Assemblée des départements de France (ADF), les Départements anticipent une perte de DMTO dès 2020 a minima de - $30 \%$ (soit - 4 Md€ par rapport à 2019). Pour mémoire, la hausse des recettes avait été continue entre 2008 et 2018, soit plus de $5 \mathrm{Md}$ ( $+6,3 \%$ en moyenne). Pour la CVAE, la perte envisagée se manifesterait en 2021 et 2022. Concernant les dépenses, la principale augmentation anticipée est celle relative au financement du RSA. Ce communiqué de I'ADF revient également sur la réforme de la taxe $d$ 'habitation et l'affectation aux conseils départementaux d'une part de TVA. II rappelle qu'à la suite de la crise de 2008, la TVA avait chuté de - 5,5\%. Ainsi, I'affectation aux départements de ressources purement cycliques (DMTO, CVAE et TVA) révèle actuellement sa dangerosité.

\section{Plan de soutien aux communes et aux départements}

Le Premier ministre a annoncé le 29 mai un plan massif de soutien aux collectivités territoriales d'un montant initial de 4,5 Md€ pour compenser les pertes liées à l'épidémie de Covid-19 en 2020 et 2021 : une compensation des pertes de recettes fiscales et domaniales de $750 \mathrm{M} €$, la prise en charge à $50 \%$ des achats de masques, un lissage des dépenses liées au Covid-19 sur trois ans, une avance sur les DTMO pour les départements et un soutien de $1 \mathrm{Md}$ à l'investissement vert. Les mesures seront insérées dans le troisième projet de loi de finances rectificative présenté en conseil des ministres le 10 juin prochain. Les catégories de collectivités bénéficiaires sont les plus impactées par la crise sanitaire : le bloc communal, les départements puis les collectivités d'outre-mer. Ce plan sera actualisé en fonction des conclusions de la mission confiée à JeanRené Cazeneuve, président de la Délégation aux collectivités territoriales et à la décentralisation de l'Assemblée nationale chargé par le Premier ministre d'une mission sur les finances locales et son montant pourra être actualisé au cours de l'année.

\section{FINANCES SOCIALES}

\section{$\pi$ Situation des compte sociaux}

\section{Un déficit sans précédent pour la Sécurité So-} ciale

La note du Haut comité du financement de la protection sociale(HCFiPS) du 15 mai constitue 
la première analyse de l'impact de la crise du COVID 19 sur les finances sociales. Pour 2019, le déficit du régime général plus le fonds de solidarité vieillesse, avec un montant de - 1,9 Md€ s'est finalement avéré à peine supérieur à celui de 2018 (-1,2Md€-meilleur résultat depuis 2002) et bien inférieur à ce qui était redouté à l'automne (- 5,4 Md€ du fait notamment de l'incidence des mesures "Gilets jaunes ») cela grâce à de meilleures rentrées de recettes. Le résultat des ASSO pour 2019 serait même en excédent de $+0,6 \%(+14,1 \mathrm{Md}$ ). De ce fait, le retour à l'équilibre de l'ensemble RG+FSV que la LFFSS avait renvoyé à 2023 (cf. Repères d'octobre 2019) pouvait légitimement être escompté pour une date plus proche. La crise économique consécutive au COVID 19 bouleverse totalement ces perspectives, avec une prévision de déficit pour I'ensemble RG+FSV de -41Md€ en 2020 (cf Repères d'avril). Celui-ci serait d'abord causé par une énorme réduction des recettes : cotisations sociales et CSG du fait d'une contraction de $-7,5 \%$ de la masse salariale ; recettes assises sur les revenus du capital ; impôts et taxes affectés à la SS (taxe sur les salaires, C3S, forfait social, TVA et autres droits sur la consommation...); en second lieu, par une sensible augmentation des dépenses dans toutes les branches : maladie (achats de matériel, rémunérations des soignants, dépenses de prévention... conduisant à un rehaussement probable de l'ONDAM de 2,4\% à $7 \%$ ), famille (augmentation des minima sociaux et du nombre de bénéficiaires), assurance chômage qui devrait enregistrer une hausse de ses dépenses de l'ordre de $11 \mathrm{Md}$ liée au chômage partiel et à l'explosion du nombre des allocataires. L'impact sur la trésorerie a conduit à un relèvement du plafond d'emprunt de I'ACOSS de 39 à $70 \mathrm{MdE}$, puis à $95 \mathrm{Md}$ le 20 mai et à une extension de $10 \mathrm{Md}$ de la garantie de l'État à I'UNEDIC. Le HCFiPS pose in fine deux question: le surcroît de dette issu de la crise COVID doit-il être considéré comme une "dette sociale » devant être reprise et amortie par la CADES ? Ne faut-il pas distinguer entre des aléas «normaux » pour la Sécurité sociale et des aléas « exceptionnels » qui ne peuvent pas être pilotés par les outils de gestion habituels?

\section{Dette sociale}

Dette sociale et « cinquième risque dépendance "

Le conseil des ministres du 27 mai a adopté deux projets de loi ordinaire et de loi organique pour assurer l'apurement de la dette sociale, qui va considérablement augmenter du fait de la crise du COVID 19, et créer un nouveau risque " dépendance ». La CADES verra sa mission-qui devait normalement s'éteindre en 2024 -prolongée jusqu'en 2033 pour assurer le remboursement d'une dette de 136 Md€ intégrant un montant de $44 \mathrm{Md} €$ de dette passée et de $92 \mathrm{Md} €$ de dette prévisible pour la période 2020-2033, sans augmentation des taux de prélèvement des ressources affectées (CSG et CRDS). Par ailleurs, une cinquième branche " dépendance » sera créée, pour laquelle seraient redéployés-à travers la Caisse nationale de solidarité pour l'autonomie - 0,15 points de CSG. La ressource supplémentaire ainsi dégagée (2,3 Md€) seraient toutefois loin du besoin supplémentaire de financement (de 6,2 à 9,2 Md€ par an) que le rapport Libault (cf. Repères de 2019) estimait indispensable pour financer la dépendance à l'horizon de 2030.

\section{त Allègement des charges et coût du travail}

\section{Débat sur une augmentation du temps de travail}

Après le Medef, une note du 5 mai de Bernard Martinot de l'Institut Montaigne a mis dans le débat public la problématique de la restauration de la capacité de production des entreprises et de leur productivité par une augmentation au moins temporaire de la durée du travail. Une telle mesure serait justifiée pour sauvegarder de nombreuses entreprises, pour faire face à de nombreux goulots de production au moment de la reprise et pour aider au redressement des finances publiques par une remise à niveau de la base des prélèvements fiscaux et sociaux. La note esquisse neuf propositions pour : assouplir les verrous à une plus grande flexibilité du travail (durée journalière et hebdomadaire maximale, gestion des RTT...) ; remettre en cause des périodes de congé (suppression du jeudi de l'Ascension comme jour férié, abandon d'une semaine de congé à Toussaint...) ; ou réduire les spécificités de la fonction publique (diminution provisoire des jours de RTT, accroissement de I'éligibilité au forfait jour, accroissement temporaire-contre rémunération- de la durée d'activité des fonctionnaires dans les secteurs en tension indispensables à la vie économique)...Ces propositions ont partagé les experts économiques, les syndicats ont réagi vivement et le gouvernement s'est cantonné dans une réserve prudente.

\section{Annulation des charges pour les TPE}

Après l'annonce du plan de déconfinement, le ministre de l'économie et des finances a confirmé le 7 mai l'annulation pure et simple des charges 
sociales patronales pour les mois de mars, avril et mai pour les TPE de moins de 10 salariés frappés par la décision de fermeture administrative. Cette mesure devrait principalement concerner les secteurs de l'hôtellerie-restauration, du tourisme, de la culture, mais non les commerces alimentaires qui sont restés ouverts, ni les grandes enseignes.

\section{Comment sortir du chômage partiel ?}

Le coût de la prise en charge du chômage partiel, qui a concerné jusqu'à 12 millions de salariés pendant la période de confinement risque de devenir insupportable pour le budget de l'État et I'UNEDIC. Le dispositif français est plus généreux que celui de ses partenaires européen (garantie de $84 \%$ du salaire net dans la limite de 4,5 SMIC contre $60 \%$ en Allemagne et un plafond de $1200 €$ en Italie), ne pousse pas à la reprise et se prête à des risques de fraude avec le recours parallèle au télétravail. Dans un entretien aux Echos du 14 mai, Philippe Martin, président du Conseil d'analyse économique évoque plusieurs pistes pour se dégager du chômage partiel, notamment: le principe d'un reste à charge pour les entreprises pour inciter à la reprise ; la réduction du plafond à 3,5 SMIC, les cadres pouvant télétravailler; une réduction progressive de la prise en charge, qui pourra être différenciée selon les secteurs, les professions les plus frappées par l'interdiction administrative (restauration, tourisme, secteur culturel...) étant celles qui devront en bénéficier le plus longtemps. Le gouvernement, qui a annoncé des contrôles assortis de sanctions pénales pour les fraudeurs et une réduction de la prise en charge à partir du 1er juin.

\section{Dépenses de santé/hôpital/ assurance maladie}

\section{Un "Ségur de la santé »}

Après la promesse du Président de la République en faveur d'un « plan massif pour l'hôpital » lors de son déplacement à Mulhouse le 26 mars, le ministre des solidarités et de la santé a annoncé, dans un entretien au JDD du 17 mai, un nouveau plan pour le système de santé, qui serait le troisième en un an après la réforme "Ma santé 2022 » et le plan pour les urgences hospitalières de l'automne 2019 (v. les Repères de 2019 sur ces sujets). Ce plan serait précédé d'une vaste concertation avec les professions et les territoires à partir du 25 mai dans le cadre d'un « Ségur de la santé » et comprendrait au moins 3 volets: un programme de revalorisation des salaires des personnels pour atteindre au moins la moyenne européenne (soit $44000 €$ par an), ce qui selon de premières estimations représenterait une enveloppe de 3 à 4 Md€ par an); une réforme du temps de travail visant à assouplir le carcan des 35 h qui conduit à des contournements coûteux ; de nouvelles organisations permettant de valoriser le travail collectif. Ces orientations ont été avalisées au conseil des ministres du 20 mai.

\section{$\lambda$ Dette sociale/dépendance /solidarité}

Une aide pourles jeunes de moins de 25 ans précaires ou modestes

Lors du débat sur la prorogation de l'état d'urgence sanitaire au Sénat le 4 mai, le Premier ministre a annoncé le versement d'une aide exceptionnelle de $200 €$ à 800000 jeunes de moins de 25 ans en situation précaire pour les aider à faire face aux conséquences financières de la crise. Cette aide serait versée début juin aux étudiants ayant perdu leur emploi ou leur stage, et à la mijuin aux jeunes de moins de 25 ans précaires ou modestes qui perçoivent les APL. Le dispositif serait pris en compte dans une nouvelle loi de finances rectificative en juin.

\section{Report de la réforme du recouvrement des pensions alimentaires}

Le ministre chargé des relations avec le Parlement a annoncé le 14 mars que le service public de recouvrement des pensions alimentaires qui, en application de la LFSS 2020, devait être mis en place dans le cadre des CAF au $1^{\text {er }}$ juin 2020 ne pourrait être mis en place du fait de la crise du COVID 19.

\section{Administrations de sécurité sociale}

\section{Une enveloppe d'1,2 Md€ pour les person-} nels des EHPAD et des hôpitaux

Dans le prolongement des mesures annoncées le mois dernier en faveur des personnels hospitaliers (cf Repères d'avril), le ministre des solidarités et de la santé a annoncé le 5 mai le versement d'une prime défiscalisée pour les personnels des établissements médico-sociaux (EHPAD...), d'un montant de $1500 €$ pour les 33 départements les plus touchés par l'épidémie et de $1000 €$ pour les autres. Le coût de cette prime pourrait s'élever à $700 \mathrm{M} €$ pour l'Assurance maladie. S'agissant des personnels hospitaliers, la prime exceptionnelle qui avait été annoncée au mois d'avril a fini par être mise en place, non sans retard, par le décret du 14 mai 2020. S'y ajoutent un financement de 
500 M€ pour faire face aux dépenses (masques, dispositifs de protection...) de ces établissements pendant la crise

\section{FISCALITÉ ET PROCÉDURE FISCALE}

TVA réduite à $5,5 \%$ sur les masques de protection et produits destinés à l'hygiène corporelle adaptés à la lutte contre la propagation du virus

Le Gouvernement a publié l'arrêté du 7 mai 2020 destiné aux personnes qui réalisent des opérations taxables à la TVA portant sur des masques de protection et des produits destinés à l'hygiène corporelle adaptés à la lutte contre la propagation du virus Covid-19 qui sont éligibles au taux réduit de $5,5 \%$ de la TVA.

Une mesure de tempérament Covid-19 concernant le dépôt à la formalité des actes concernant la vie des sociétés

Les services de l'enregistrement acceptent temporairement que le dépôt des actes des sociétés soit effectué par voie dématérialisée (actes constatant l'augmentation, l'amortissement ou la réduction de capital d'une société ou les actes constatant la transformation d'une société, actes de prorogation ou de dissolution d'une société). Si des droits sont dus, seul le virement est envisageable. Cette mesure de tempérament s'applique jusqu'au 10 juillet 2020.

\section{COMPTABILITÉ PUBLIQUE}

\section{त Réglementation comptable}

Trésorerie de l'État : extension des dépôts obligatoires au Trésor

Afin d'améliorer la gestion de la trésorerie de l'État, le projet de loi portant « diverses dispositions liées à la crise sanitaire, à d'autres mesures urgentes ainsi qu'au retrait du Royaume-Uni de I'Union européenne », présenté au conseil des ministres du 7 mai, prévoit dans son article 4 que le gouvernement pourra, par ordonnances de l'article 38 de la Constitution, prescrire le dépôt au trésor public des disponibilités des personnes morales soumises aux règles de la comptabilité publique et des organismes publics ou privés chargés d'une mission de service public. Cette faculté d'extension des organismes tenus de déposer leurs fonds au trésor est ouverte pour un période d'un an.

\section{MANAgement PUBlic}

\section{$\eta$ Organisation administrative \\ Le rapport de Jean Castex}

Le rapport de Jean Castex, délégué interministériel, coordonnateur de la stratégie nationale de déconfinement, a été publié le 12 mai, juste après la première phase. Bel exemple de gestion publique de crise.

\section{त Administration numérique}

Stop Covid : imparfait mais national

La mise au point de l'application Stop Covid, qui vise à informer les personnes testées positives des personnes avec lesquelles elles ont été en contact et à qui elles auraient pu transmettre le virus, (v. Repères d'avril 2020) est plus difficile que prévu. Apple a refusé de débrider Bluetooth : le détecteur de contact ne pourra donc pas fonctionner en permanence. Par ailleurs, des pays voisins, dont l'Allemagne, se tournent vers le procédé proposé par l'alliance Google-Apple qui repose sur un stockage "décentralisé » des données sur les téléphones qui, selon les experts français, offre moins de sécurité que le stockage « centralisé » préconisé par I'INRIA et ses partenaires. L'Inria a tenté de mettre au point un protocole de synthèse «Desire ». Le 13 mai, la Commission a recommandé l'interopérabilité transfontalière des dispositifs de traçage et a publié un guide à cet effet. Selon, un sondage Harris interactive pour DataPublica (1 051 répondants en ligne, le 7 mai) : $59 \%$ des français interrogés seraient favorables à la mise en œuvre de l'application Stop covid ; $49 \%$ auraient I'intention de l'installer sur leur téléphone mais $54 \%$ n'ont pas confiance dans le Gouvernement à propos de l'utilisation du fichier centralisant les données. La CNIL a donné son accord au projet par une délibération du 25 mai. Les deux assemblées qui en ont débattu le 27 mai ont donné leur accord après que le gouvernement a réaffirmé l'utilité du dispositif pour détecter et stopper les reprises du virus et appelé les garanties du système du point de vue de la protection des libertés individuelles (volontariat, anonymat, conservation temporaire des données). Un dossier de presse a été publié. Le traitement informatiqe a fait l'objet du décret $n^{\circ}$ 2020-650 du 29 mai 2020, JORF du 30 mai, texte $n^{\circ} 17$.Stop Covid devrait être disponible le 2 juin.

\section{Fichier des personnes positives et contacts}

L'article 11 de la loi n²020-546 du 11 mai 2020, prolongeant l'état d'urgence et complétant ses dispositions, publiée au JORF du 12 mai, texte 
$n^{\circ} 1$, et le décret $n^{\circ} 2020-567$ du 14 mai, publié au JORF du 15 mai, texte $n^{\circ} 10$, organisent les fichiers et les traitements relatifs au dépistage des « patients zéro » et à l'identification de leurs « contacts » pour organiser le dépistage et briser les chaînes de contamination. Un fichier Contact Covid, créé à partir d'Amelipro, sera géré par la CNAM pour recenser les « cas contact». Un fichier SI-Dep sera géré par I'APHP pour centraliser les résultats des dépistages. Le décret définit les personnes responsables du traitement, les finalités, les données recueillies, la durée de conservation, l'exercice des droits individuels conformément à la décision du Conseil constitutionnel n²020-800 DC du 11 mai 2020 (§ 59 à78).

\section{Attestation numérique}

Un modèle numérique de la nouvelle déclaration de déplacement hors de son département et dans un rayon de plus de $100 \mathrm{~km}$ a été mis en ligne le 12 mai, plus rapidement que celui de la première attestation (cf. Repères d'avril).

\section{La plateforme d'innovation numérique du} ministère de la santé

La plateforme e-genius, le guichet national de I'innovation et des usages en e-santé, a été ouvert le 13 mai par le ministère des solidarités et l'agence du numérique en santé. Elle a pour but de faire émerger des projets en santé numérique.

\section{$\lambda$ Fonction publique}

\section{Un guide du déconfinement}

La DGAFP a publié un document questions/réponses qui traite des problèmes posés à l'occasion du retour vers une activité normale dans la fonction publique à partir du 11 mai.

\section{EUROPE}

\section{$\pi$ Politique européenne : semestre euro- péen}

De sombres prévisions économiques de printemps

Le 6 mai 2020, la Commission a publié ses prévisions économiques de printemps anticipant " une récession profonde et inégale » et "une reprise incertaine ». La Commission table en effet sur une contraction de la zone euro record de $73 / 4 \%$ en 2020 , et une croissance de $61 \% 4 \%$ en 2021 et, pour l'ensemble de I'Union, un retrait de $71 / 2 \%$ en 2020 et une progression de $6 \%$ en 2021, soit une baisse d'environ 9 points de pourcentage par rapport aux prévisions de l'automne 2019. Par ailleurs, ce choc est symétrique dans la mesure où tous les États membres ont été touchés par la pandémie, mais la baisse de la production en 2020 (de $-4 \frac{1}{4} \%$ en Pologne à $-93 / 4 \%$ en Grèce), comme la vigueur du rebond en 2021, devraient être très hétérogènes. Sans surprise Le taux de chômage de la zone euro devrait augmenter, passant de 7,5\% en 2019 à 91/2\% en 2020, avant de retomber à $81 / 2 \%$ en 2021 . Le taux de chômage de l'ensemble de l'UE devrait aussi être en hausse, passant de 6,7 \% en 2019 à $9 \%$ en 2020, avant de redescendre à environ $8 \%$ en 2021.

\section{Recommandations pour une réaction coor-} donnée à la pandémie

Le 20 mai, la Commission a proposé dans le cadre du Semestre européen des recommandations par pays qui fournissent à tous les États membres de I'UE des orientations en matière de politiques économiques dans le contexte de la pandémie de coronavirus et mettent l'accent sur les problèmes les plus urgents posés par la pandémie ainsi que sur la relance d'une croissance durable.

\section{$\pi$ Politique européenne : plan de relance}

Accord de la zone euro sur les premières mesures anti-crise

Le 8 mai 2020, lors d'une réunion de l'Eurogroupe, les dix-neuf ministres des finances de la zone euro se sont entendus sur les détails d'une première riposte à la crise, mais tout reste à faire sur un grand plan de relance. Le Mécanisme européen de stabilité (MES), le fonds de sauvetage de la zone euro né en 2012, pourra ainsi mettre à la disposition des pays les plus touchés, pour des dépenses liées au virus et sans autres conditions, des lignes de crédits « de précaution», pouvant atteindre jusqu'à $2 \%$ de leur produit intérieur brut (PIB).

\section{Proposition franco-allemande d'un plan de relance de $500 \mathrm{Md} €$}

Contre toute attente, le 18 mai 2020, La France et l'Allemagne proposent un plan de relance européen de 500 milliards d'euros qui pourrait comporter une forme de mutualisation des dettes. Le 21 , les « quatre frugaux » de l'Europe s'opposent au plan de relance franco-allemand

\section{Le plan de relance audacieux d'Ursula von der Leyen}

Le 27 mai, la Commission présentait une communication sur vaste projet de plan de relance pour l'Europe qui repose sur plusieurs piliers: soutien aux États-membres dans le domaine des investissements et des réformes pour faire face à la crise ; relance de l'économie en stimulant l'investissement privé; leçons de la crise. Le plan 
fondé sur l'instrument de relance « Next Generation EU » et sur une refonte du budget de I'Union d'un montant de 1100 Md€ entre 2021 et 2027. Il comporte de nombreuses propositions innovantes : le plan de soutien aux pays les plus touchés par la crise sanitaire transiterait par le budget de l'Union; le plafond de celui-ci serait relevé à $2 \%$ du revenu national brut de l'Union ; les 750 Md€ sur trois ans seraient répartis entre $500 \mathrm{Md} €$ de subventions et $250 \mathrm{Md}$ de prêts ; les soutiens aux États seraient conditionnés par une validation collective des États-membres; les aides seraient distribuées aux États pour soutenir les investissements et les réformes nécessaires à une reprise durable (560 Md€), aider rapidement les secteurs les plus touchés (React-Union $55 \mathrm{Md€}$ ), soutenir la transition écologique, développer l'investissement, amorcer une Europe de la santé ; la Commission emprunterait elle-même sur les marchés avec la garantie des États-membres ; ces emprunts seraient remboursés à partir de 2028 et avant 2058 par le budget de l'Union au moyen des contributions des États-membres ou de nouvelles ressources propres reliées au Pacte vert: inclusion du transport maritime et aérien dans le système d'échange de quotas d'émissions polluantes, taxe carbone aux frontières de I'Union, taxe sur les plastiques à usage unique, taxe sur les bénéfices des multinationales du numérique. II n'est pas certain que cette amorce d'une " union de transfert » soit acceptée par l'unanimité des chefs d'État et de gouvernement des Étatsmembres dans les prochaines semaines.

\section{Aide d'État : souplesse toujours de la Com- mission}

Comme au mois d'avril, la Commission européenne a poursuivi son application allégée des règles relatives aux aides d'État en acceptant des mesures prises dans tous les États membres sans exception. Dans une démarche plus générale, le 11 mai, elle a invité les États membres à se prononcer sur sa proposition mise à jour visant à exempter de l'examen préalable les aides octroyées au moyen de fonds nationaux à des projets soutenus dans le cadre de certains programmes gérés de manière centralisée par I'Union.

\section{Pacte de stabilité}

\section{Christine Lagarde pour une révision des} termes du pacte de stabilité

Le 18 mai, interrogée par plusieurs médias européens, la présidente Christine Lagarde a déclaré que le Pacte de stabilité et de croissance, aujourd'hui suspendu par la Commission « ne de- vrait pas être remis en place tel qu'il était avant la crise ». Elle suggère de s'inspirer de plusieurs propositions, notamment celles du FMI, qu'elle dirigeait auparavant, pour assouplir ce cadre budgétaire européen.

\section{$\lambda$ Euro}

\section{BCE : le PEPP menacé par la Cour de Karls- ruhe?}

La décision du 5 mai 2020 du Tribunal constitutionnel allemand critiquant le rachat par la BCE de dettes publiques concerne les mesures prises pour répondre à la crise de 2008 (voir cette chronique « hors covid » de mai 2020 dans ce même numéro) pourrait avoir des conséquences sur le plan de relance de la BCE (Pandemic Emergency Purchase Programme: PEPP). L'absence de soutien apporté par la chancelière Merkel aux juges de Karlsruhe en limite la portée même si la question de la participation de la Bundesbank aux prochaines opérations reste pendante.

\section{Fiscalité}

\section{Retard à prévoir pour deux directives fis- cales européennes}

Le 27 mai, en raison de la crise sanitaire, il a été constaté que les États membres n'étaient pas prêts pour l'entrée en vigueur de deux directives, celle sur les montages fiscaux et celle relative à la TVA sur les ventes à distance. Leur application a été décalée de trois à six mois.

\section{INTERNATIONAL}

\section{Un rapport de l'OCDE sur les politiques contre la pandémie}

Le 4 mai 2020, I'OCDE a organisé un Webinaire « OECD Tax Talks » pour faire le point sur ses travaux relatif à la fiscalité dans le cadre du Covid19. Cette réunion prolonge les résultats des collectes de données de l'OCDE qui avaient donné lieu le 15 avril 2020 à un rapport mettant en valeur le rôle fondamental des mesures fiscales et budgétaire pour soutenir les ménages et les entreprises pendant la période de confinement, puis renforcer la reprise.

\section{L'OCDE reporte le projet de taxation des multinationales du numérique}

Le 4 mai, l'OCDE a annoncé que, sous l'effet de la pandémie, le projet finalisé de " taxe GAFA » mondiale, nouvel impôt mondial a vocation à remplacer rapidement les taxes GAFA nationales ne serait finalement présenté qu'en octobre au G20 finances de Washington. 


\section{La Cour des comptes néerlandaise critique les aides à AirFrance-KLM}

La Cour des comptes d'Amsterdam a déclaré mercredi 20 mai 2020 que la décision prise en 2019 par l'État néerlandais d'entrer à hauteur de $14 \%$ dans le capital d'Air France-KLM (afin de détenir une participation identique à celle de la République française et de peser sur les décisions du groupe de transport aérien) était illégale car le gouvernement aurait dû informer au préalable le Parlement de son initiative. Cependant la Cour ne demande pas d'annuler l'opération mais elle formule une série de recommandations, invitant notamment le gouvernement à éviter qu'une telle décision ne se reproduise à l'avenir.
Retrouvez sur www.gestionfinancespubliques. info :

- I'intégralité des REPÈRES d'actualité des finances et de la gestion publiques depuis 2015

-le 15 du mois, les REPÈRES d'actualité du mois précédent

-le 15 janvier 2020, le BEST OF des repères d'actualité de l'année 2019.

- et, en continu, les réflexions des blogueurs de G\&FP.
Aurélien BAUDU (fiscalité et procédure fiscale),

Fabrice BIN (Europe, international), Jean-François CALMETTE (budget de l'État et des opérateurs),

Matthieu HOUSER (finances locales), Michel LE CLAINCHE (comptabilité publique, management public, coordination), Yves TERRASSE (finances sociales). 